CASE REPORT

\title{
Extubation failure due to phrenic nerve injury
}

\section{O Williams, A Greenough, N Mustfa, S Haugen, G R Rafferty}

Arch Dis Child Fetal Neonatal Ed 2003;88:F72-F73

A 26 week gestation infant had an increasingly elevated right hemidiaphragm following drainage of bilateral pleural effusions and failed extubation on numerous occasions. Electric stimulation of the phrenic nerves revealed absent activity on the right, indicating phrenic nerve injury from chest tube drain insertion. Diaphragmatic plication was performed and the infant successfully extubated four days later.

$\mathrm{E}$ xtubation failure occurs in approximately 33\% of premature infants. This is usually the result of immature respiratory control or an imbalance between respiratory muscle strength and respiratory load. The diaphragm is the main respiratory muscle in neonates and thus phrenic nerve injury, although rare, can also cause respiratory embarrassment.

\section{CASE REPORT}

A 26 week gestation, male infant, birth weight $1024 \mathrm{~g}$, responded well to surfactant administration and had minimal ventilatory requirements by day seven. Chest radiographs confirmed the initial diagnosis of respiratory distress syndrome (RDS) and that he had normally positioned hemidiaphragms. On day 10, the baby had increasing ventilatory requirements, acidosis, and hypotension. The infant became increasingly oedematous and developed bilateral pleural effusions, which were drained on day 33 using size 10F Argyle catheters using a technique of accepted standards (figure la). Subsequent chest radiographs demonstrated an increasingly elevated right hemidiaphragm (figure $\mathrm{lb}$ ), a right phrenic nerve injury was suspected. Unfortunately, despite minimal ventilatory requirements the infant failed at least four trials of extubation over the subsequent weeks. During this period, his lung volume, assessed by measurement of the functional residual capacity, was $20 \mathrm{ml} / \mathrm{kg}$ (normal range 24 to $36 \mathrm{ml} / \mathrm{kg}$ ). Transdiaphragmatic crying pressures were $20 \mathrm{cmH}_{2} \mathrm{O}$ (the expected value an infant of this gestation is $40 \mathrm{cmH}_{2} \mathrm{O}^{1}$ ). Ultrasound examination revealed a markedly hypokinetic right diaphragm. Percutaneous electric stimulation of the phrenic nerves was performed using bipolar electrodes placed over the sternomastoid muscle. Reproducible responses could only be obtained on the left (figure 2), confirming the diagnosis of right phrenic nerve injury with no recovery. The infant underwent a thoracotomy and plication of the right hemidiaphragm on day 75. He was successfully extubated on the fourth postoperative day. After three days of nasal continuous positive airways pressure, he subsequently required only minimal amounts of supplementary oxygen via nasal cannulae.

\section{DISCUSSION}

Chest drain insertion in infants has been associated with a number of complications. In an early series, direct perforation of the lung has been reported to occur in up to $25 \%$ of cases,
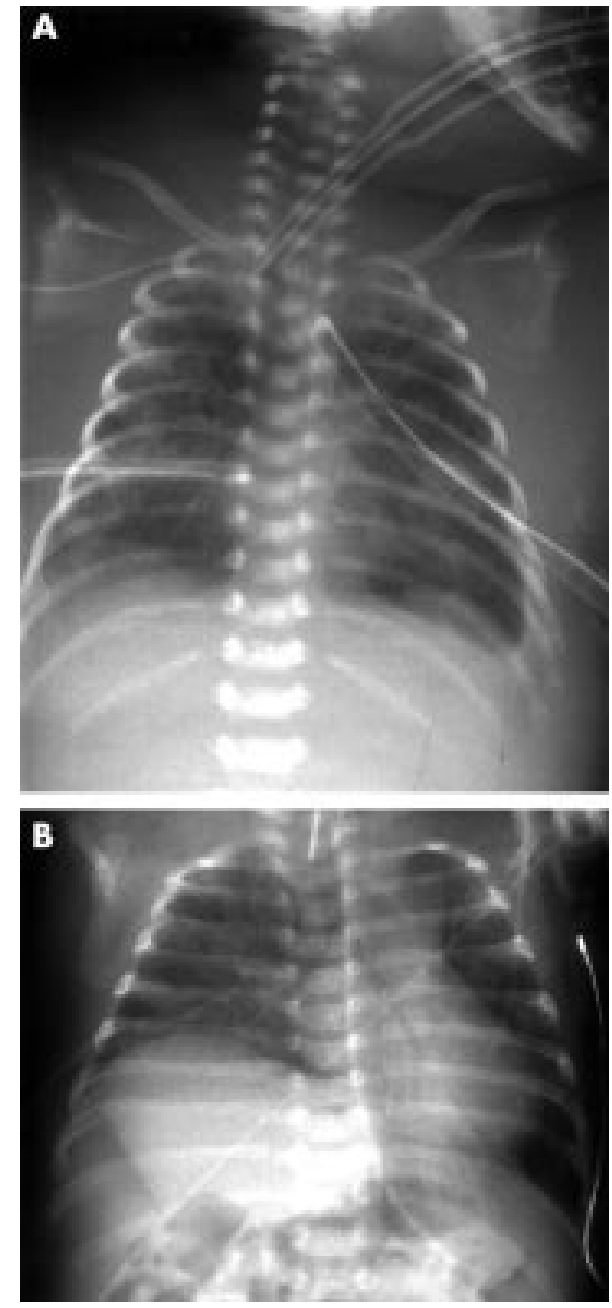

Figure 1 (A) Chest radiograph taken two days after bilateral chest drain placement. The right chest drain tip abuts the mediastinum. (B) Chest radiograph taken 38 days later demonstrates a markedly elevated right diaphragm.

this may lead to a bronchopleural fistula. ${ }^{2}$ Trauma to the thoracic duct at the posterosuperior mediastinum may result in the development of a chylothorax. ${ }^{3}$ Haemorrhagic pericardial effusion, causing cardiac tamponade has also been reported following chest drain insertion. ${ }^{4}$ Very rarely phrenic nerve injury in neonates, which can occur following birth trauma or cardiothoracic surgery, results from chest drain placement. ${ }^{56}$ Phrenic nerve injury is most likely to occur if the drain is placed deep in the chest where the phrenic nerve runs over the mediastinum. In the infant we report, the most likely cause was direct nerve injury cause by the chest drain tip (figure la). Although both chest drains abut the mediastinum (figure la), we speculate the left drain was in a different position with respect to the phrenic nerve and hence no injury was caused. 


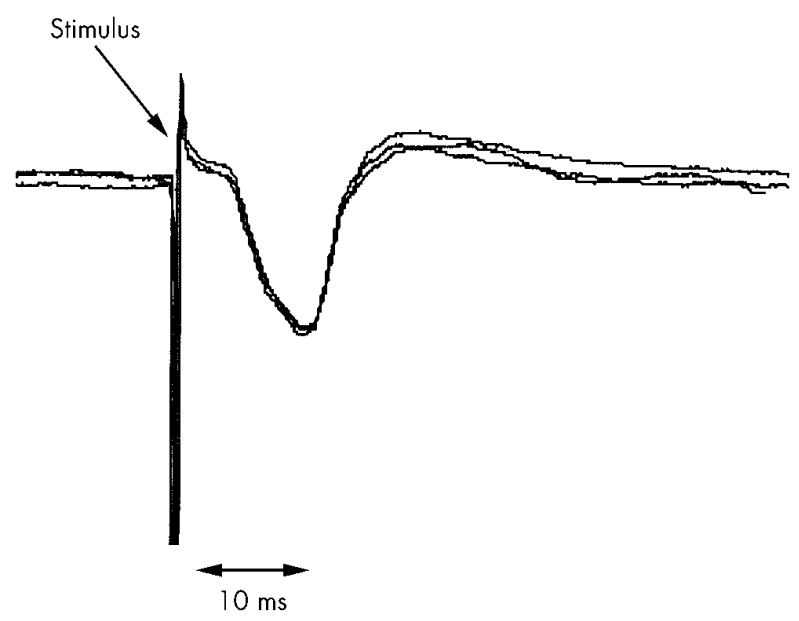

Figure 2 Electromyographic (EMG) waveform following left unilateral phrenic nerve stimulation. After the stimulus artefact (marked stimulus) three superimposed EMG compound muscle action potential traces are shown. The mean phrenic nerve conduction time was 5.4 milliseconds. On right phrenic nerve stimulation only the stimulus artefact was present.

Chest drains allowed to remain across the mediastinum have even been reported to cause pressure necrosis of the oesophagus. ${ }^{7}$ The impact of the phrenic nerve damage and the consequent raised right hemidiaphragm is demonstrated by the low lung volume of the infant. In addition, the crying transdiaphragmatic pressures were approximately $50 \%$ of expected. ${ }^{1}$ One explanation for the poor respiratory muscle function could be disuse atrophy resulting from the prolonged ventilation. We, however, suggest that the low pressures generated were the result of the right phrenic nerve abnormality, as three days following diaphragmatic plication the infant was successfully extubated.

Diagnosis of phrenic nerve injury is often delayed due to the rarity of the complication and the lack of appropriate, readily available tests. Early diagnosis is particularly difficult in ventilated patients, as the radiographic appearances may be obscured by the effects of positive pressure ventilation. The commonest investigation is ultrasound examination of the diaphragm, but this only provides a qualitative assessment and does not discriminate between diaphragmatic and phrenic nerve dysfunction. The latter condition can be diagnosed by direct stimulation of the phrenic nerves and demonstration of prolonged phrenic nerve latency or an absent signal. The technique has been shown of value in the postoperative management of children who have undergone cardiothoracic procedures ${ }^{8}$ but, to our knowledge, has not been used before to diagnose the cause of extubation failure in a preterm infant.

A high proportion of infants, $72 \%$ in one series, ${ }^{9}$ with phrenic nerve injury due to obstetric or operative trauma require surgical plication of the diaphragm. Following plication improvement is usually rapid, as in our patient. In 50 patients aged between 4 days and 7 years the mean time for withdrawal of ventilatory support postoperatively was three days (range 0 to 6 ). ${ }^{7}$ Such data argue for early intervention and hence avoidance of prolonged ventilation with the associated increased risk of complications such as nosocomial pneumonia. ${ }^{10}$ Spontaneous recovery is unlikely if there are persistent signs of phrenic nerve palsy one month post insult.' In this infant, use of electric stimulation and demonstration of an absent response from the right phrenic nerve confirmed the lack of recovery and thus determined the timing of the surgical intervention.

\section{ACKNOWLEDGEMENTS}

Dr Olivia Williams is supported by the Children Nationwide Medical Research Fund. We thank Ms Sue Williams for secretarial assistance.

\section{Authors' affiliations}

O Williams, A Greenough, N Mustfa, S Haugen, G R Rafferty, Children Nationwide Regional Neonatal Intensive Care Centre, King's College Hospital, London SE5 9RS, UK

Correspondence to: Professor Anne Greenough, Children Nationwide Regional Neonatal Intensive Care Centre, King's College Hospital, London SE5 9RS, UK; anne.greenough@kcl.ac.uk

Accepted 2 May 2002

\section{REFERENCES}

1 Scott CB, Nickerson BG, Sargent CW, et al. Developmental pattern of maximal transdiaphragmatic pressure in infants during crying. Pediatr Res 1983;17:707-9.

2 Moessinger AC, Driscoll JM, Wigger HJ. High incidence of lung perforation by chest tube in neonatal pneumothorax. J Pediatr 1978:92:635-7.

3 Kumar SP, Belik J. Chylothorax-a complication of chest tube placement in a neonate. Crit Care Med 1984;12:411-12.

4 Quak JME, Szatmari A, van den Anker JN. Cardiac tamponade in a preterm neonate secondary to a chest tube. Acta Paediatr 1993;82:490-1.

5 Marinelli V, Ortiz A, Alden E. Acquired eventration of the diaphragm: a complication of chest tube placement in neonatal pneumothorax. Pediatrics 1981;67:552-4.

6 Ayalon A, Anner H, Moghilner M, et al. Eventration of the diaphragm due to phrenic nerve injury caused by intercostals drainage. J Pediatr Surg 1979;14:473-4.

7 Cairns PA, McClure BG, Halliday HL, et al. Unusual site for oesophageal perforation in an extremely low birthweight infant. Eur J Pediatr 1999:158:152-3.

8 Russell RI, Mulvey D, Laroche C, et al. Bedside assessment of phrenic nerve function in infants and children. J Thorac Cardiovasc Surg 1991;101:143-7.

9 de Vries TS, Koens BL, Vos A. Surgical treatment of diaphragmatic eventration caused by phrenic nerve injury in the newborn. J Pediatr Surg 1998;33:602-5.

10 Tsugawa C, Kimura K, Nishijima E, et al. Diaphragmatic eventration in infants and children: is conservative treatment justified? J Pediatr Surg 1997;32:1643-4. 